\title{
Effects of a DPP4 inhibitor on cisplatin-induced acute kidney injury: study protocol for a randomized controlled trial
}

\author{
Seon Ha Baek', Se Hyun Kim², Jin Won Kim², Yu Jung Kim², Keun-Wook Lee² and Ki Young Na ${ }^{1 *}$
}

\begin{abstract}
Background: Cisplatin is a potent chemotherapeutic agent, but its nephrotoxicity, which results in acute kidney injury (AKI), often limits its clinical application. Although many studies have attempted to target the mechanism responsible for its nephrotoxicity, no such method has been demonstrated to be effective in clinical trials. Recently, a dipeptidyl peptidase-4 (DPP4) inhibitor has been reported to have a renoprotective effect in a mouse model of cisplatin-induced AKI. Therefore, we will evaluate whether a DPP4 inhibitor protects the kidney from cisplatin-induced injury in humans.

Methods/Design: This is a single-center, prospective, randomized, double-blind, placebo-controlled trial. A total of 182 participants who are scheduled for cisplatin treatment will be enrolled and randomly assigned to receive either a DPP4 inhibitor (gemigliptin) or a placebo. Participants will take the study drugs for 8 days starting 1 day before cisplatin treatment. The primary outcome of interest is the incidence of AKI at 7 days after finishing treatment with cisplatin. The secondary outcomes include changes in serum creatinine levels and estimated glomerular filtration rates from baseline to 7 days after cisplatin treatment.
\end{abstract}

Discussion: This is the first clinical trial to investigate the effect of a DPP4 inhibitor on cisplatin-induced AKI. Trial registration: ClinicalTrials.gov number NCT02250872, December 26, 2014.

Keywords: Acute kidney injury, Cisplatin, DPP4 inhibitor, Nephrotoxicity

\section{Background}

Cisplatin is a potent chemotherapeutic agent that is approved for the treatment of several types of cancer, including bladder cancer, cervical cancer, non-small cell lung cancer, and squamous cell carcinoma of the head and neck [1]. However, the kidneys are particularly affected by cisplatin because it accumulates there [1]. Cisplatin nephrotoxicity is manifested by hypokalemia, hypomagnesemia, and acute kidney injury (AKI), which often limit its clinical use [1-4]. Impairment of renal function is common and dose-dependent. AKI is found in 6-35\% of patients treated with a single dose of cisplatin and in $50-75 \%$ of patients treated with multiple doses [5-7].

\footnotetext{
* Correspondence: kyna@snubh.org

'Division of Nephrology, Department of Internal Medicine, Seoul National University Bundang Hospital, 82, Gumi-ro 173 Beon-gil, Bundang-gu, Seongnam, Gyeonggi-do 463-707, South Korea

Full list of author information is available at the end of the article
}

Abnormal renal hemodynamics and renal tubular cell toxicity resulting from oxidative stress, apoptosis, and inflammation are considered to be the mechanisms of cisplatin-induced nephrotoxicity [1, 8-14]. Different strategies targeting each mechanism of nephrotoxicity have been evaluated for their abilities to prevent and/or attenuate renal injury $[1,15,16]$. However, none of these strategies has been demonstrated to be effective in clinical trials [15].

Dipeptidyl peptidase-4 (DPP4) inhibitors are currently used in the treatment of type 2 diabetes mellitus to improve glucose tolerance by increasing the half-lives of glucagon-like peptide-1 (GLP-1) and glucose-dependent insulinotropic peptide $[17,18]$. However, in addition to the glucose-lowering effects of DPP4 inhibitors, tissueprotective effects of DPP4 inhibition have been also demonstrated [19-23]. In particular, studies have shown that DPP4 inhibitors can protect the kidney from diabetic nephropathy, ischemia-reperfusion injury, and 
chronic kidney disease [24-28]. Recently, Kataqiri et al. have reported that a DPP4 inhibitor has a renoprotective effect in rodent cisplatin-induced AKI models by enhancing GLP-1 signaling [29]. Based on these reports of the effects of DPP4 inhibitors, we hypothesize that treatment with a DPP4 inhibitor will have a beneficial effect in cisplatin-induced AKI.

Therefore, we will conduct this clinical trial in patients treated with cisplatin.

\section{Methods/design \\ Hypothesis}

Treatment with a DPP4 inhibitor will prevent and/or ameliorate cisplatin-induced AKI in humans. Compared with placebo-treated patients, the incidence of AKI will be lower in DPP4 inhibitor-treated patients.

\section{Study design}

This is a single-center, prospective, randomized, doubleblind, placebo controlled study. This study is an investigator-initiated clinical trial. The study algorithm is described in Fig. 1. After enrollment, clinical follow-up will be performed 7 days after cisplatin treatment.

\section{Study participants and measurements}

Cancer patients aged 18-70 years treated with intravenous cisplatin will be screened. The following will be conducted at the initial visit: (1) a questionnaire regarding active cancer history, chemotherapy, medical history, and history of nephrotoxic use including the use of nonsteroidal anti-inflammatory drugs (NSAIDs), antibiotics, contrast media, and calcineurin inhibitors; (2) a physical examination of all systems; (3) height and weight measurements; (4) blood pressure and pulse rate measurements. Participants who meet all of the inclusion and exclusion criteria and provide written, informed consent are eligible for this study (Table 1).

Serum creatinine ( $\mathrm{SCr}$ ) will be measured by the isotope dilution mass spectrometry-traceable method using a Toshiba TBA 200FR Analyzer (Toshiba, Tokyo, Japan). The estimated glomerular filtration rate (eGFR) will be calculated using the Chronic Kidney Disease Epidemiology Collaboration equations (CKD EPI). The CKD EPI formula, expressed as a single equation, is eGFR $=141 \times$ $\min (\mathrm{SCr} / \mathrm{\kappa}, 1)^{\alpha} \times \max (\mathrm{SCr} / \kappa, 1)^{-1.209} \times 0.993^{\mathrm{Age}} \times 1.018$ [if female] $\times 1.159$ [if black], where $\mathrm{K}$ is 0.7 for females and 0.9 for males, $\alpha$ is -0.329 for females and -0.411 for males, min indicates the minimum of $\mathrm{SCr} / \mathrm{k}$ or 1 , and $\max$ indicates the maximum of $\mathrm{SCr} / \mathrm{\kappa}$ or 1 [30] .

\section{Randomization}

A research coordinator will conduct the randomization and deliver the study drug. The participants and investigators will be blinded to the treatment assignment. A list of random numbers will be generated by an independent statistician. Eligible participants will be randomly assigned 1:1 to either the treatment group or the control group in accordance with the predefined randomization list with a block size of four. The randomization will be stratified on the basis of the number of times cisplatin is




Table 1 Inclusion and exclusion criteria

\begin{tabular}{ll}
\hline Inclusion criteria & Exclusion criteria \\
\hline Age between 18 and 70 & Diabetes mellitus \\
& Chronic kidney disease stage \\
& V-V (eGFR $\left.<30 \mathrm{ml} / \mathrm{min} / 1.73 \mathrm{~m}^{2}\right)$ \\
& History of transplantation \\
& History of acute kidney injury \\
& before randomization \\
& Active infection \\
& Use of other nephrotoxic agents \\
Cancer patients treated & such as nonsteroidal anti-inflammatory \\
with intravenous cisplatin & drugs, aminoglycosides, colistin, \\
& vancomycin, cyclosporine, tacrolimus \\
& Receiving contrast media during last $72 \mathrm{~h}$ \\
& Liver disease (bilirubin $>2$ mg/dl, \\
& transaminase levels $>2.5$ times the \\
& upper limit normal) \\
& Patients with high risks of dehydration \\
& owing to poor oral intake \\
& High blood pressure (>180/110 mmHg \\
& despite antihypertensive medications) \\
& Hypersensitivity to gemigliptin or its \\
excipients & Low compliance to gemigliptin treatment \\
Written consent & \\
&
\end{tabular}

Abbreviation: eGFR estimated glomerular filtration rate

administered (one or more than two) and on the cisplatin dose $\left(<\right.$ or $\left.\geq 50 \mathrm{mg} / \mathrm{m}^{2}\right)$ and will utilize a randomized block design.

\section{Treatments}

A selective DPP4 inhibitor, gemigliptin, which is clinically available, will be used in this study. The gemigliptin and placebo tablets will be provided by LG Life Sciences (Seoul, Korea). After randomization, the participants will take either a gemigliptin (treatment group) or placebo (control group) pill for 8 days starting 1 day before cisplatin treatment. The tablet shapes and packaging of the placebo pills are identical to those of the gemigliptin pills. The prescription and administration of the study drugs will be conducted in a double-blind manner. Based on a study that reported a renoprotective effect of a DPP4 inhibitor in a mouse model of cisplatin-induced AKI [29], $100 \mathrm{mg} /$ day of gemigliptin will be administered to the participants in two divided doses for 8 days starting 1 day before cisplatin treatment (Fig. 2). The administration of other nephrotoxic drugs, such as NSAIDs, aminoglycosides, colistin, and vancomycin, will be prohibited during the study, and exposure to contrast media during the $72 \mathrm{~h}$ prior to treatment will result in exclusion from the study. Investigators or research coordinators will evaluate drug compliance by counting pills, and participants with less than $80 \%$ compliance will be removed from the study.

\section{Chemotherapy regimen and precautions}

A hydration protocol will be identically applied to both the treatment and control groups. Cisplatin will be mixed with $500 \mathrm{ml}$ of isotonic saline and will be infused into each patient over $30 \mathrm{~min}$. Participants treated with $\geq 50 \mathrm{mg} / \mathrm{m}^{2}$ of cisplatin will receive $2,000 \mathrm{ml}$ of $0.45 \%$ saline and $100 \mathrm{ml}$ of $15 \%$ mannitol. Participants treated with $<50 \mathrm{mg} / \mathrm{m}^{2}$ of cisplatin will receive $1,000 \mathrm{ml}$ of $0.45 \%$ saline (Table 2 ).



Fig. 2 Study schedule. Abbreviations: $R$, randomization; $W t$, weight; $H t$, height; $B P$, blood pressure; $C B C$, complete blood count; $A S T$, aspartate aminotransferase; $A L T$, alanine aminotransferase; chol, cholesterol; $B \cup N$, blood urea nitrogen; $C r$, creatinine; $t \mathrm{CO}_{2}$, total $\mathrm{CO}_{2} ; U / A$, urinalysis; DPP4, dipeptidyl peptidase 4 
Table 2 Hydration protocol

\begin{tabular}{lll}
\hline Cisplatin dose & $\begin{array}{l}\text { Before and after cisplatin } \\
\text { infusion }\end{array}$ & During cisplatin infusion \\
\hline$\geq 50 \mathrm{mg} / \mathrm{m}^{2}$ & $\begin{array}{l}2,000 \mathrm{ml} \text { of } 0.45 \% \text { saline with } \\
100 \mathrm{ml} \text { of } 15 \% \text { mannitol }\end{array}$ & $\begin{array}{l}\text { Mix cisplatin in } 500 \mathrm{ml} \\
\text { of isotonic saline }\end{array}$ \\
$<50 \mathrm{mg} / \mathrm{m}^{2}$ & $1,000 \mathrm{ml}$ of $0.45 \%$ saline & $\begin{array}{l}\text { Mix cisplatin in } 500 \mathrm{ml} \\
\text { of isotonic saline }\end{array}$ \\
\hline
\end{tabular}

\section{Outcome measures}

The primary outcome of interest is the incidence of AKI at 7 days after cisplatin treatment, where AKI is defined as any of the following: an increase in SCr levels of $\geq 0.3$ $\mathrm{mg} / \mathrm{dl}$, an increase in SCr levels of $50 \%$ compared with baseline levels, or a decrease in the eGFR by $\geq 25 \%$ compared with baseline [31-33]. The secondary outcomes will include changes in SCr levels and the eGFR compared with baseline values for 7 days after cisplatin treatment.

\section{Clinical and laboratory evaluations}

The physical examination and medication reviews will be conducted before and after 8 days of treatment. Laboratory evaluations will be performed twice, before and after 8 days of treatment; the laboratory evaluations will include a complete blood count; measurements of the levels of hemoglobin, electrolytes, SCr, calcium, phosphorous, protein, albumin, and glucose; a liver function test; and urinalysis. DPP4 activity and the concentration of the DPP4 inhibitor will be measured in serum and plasma after treatment.

\section{Safety issues}

Any occurrence of headache, diarrhea, upper respiratory tract infection, hypoglycemia, or hypersensitivity reaction will be recorded during treatment with gemigliptin/ placebo. All adverse events, including serious adverse events, will be recorded and followed up during the study period or until resolution. All serious adverse events will be graded and reported to investigators and the ethics committee.

\section{Sample size calculations}

No previous study has evaluated the effect of a DPP4 inhibitor on cisplatin-induced AKI. We expect that the incidence of AKI will be $25 \%$ in patients treated with a single dose of cisplatin and that treatment with a DPP4 inhibitor could reduce this incidence by $60 \%$. We calculated the required sample size for an estimated dropout rate of $20 \%$, a one-sided level of significance of $\alpha=5 \%$, and a power of $80 \%$ and found that 91 participants will be needed in each group to find a significant difference using a $X^{2}$ test. A total of 182 participants will be included in the analysis.

\section{Statistical analyses}

Statistical analyses will be conducted on both per protocol (PP) and intention-to treat (ITT) bases. For the PP analysis, all participants who completed the study will be included to evaluate the primary and secondary outcomes. For the ITT analysis, all participants who were enrolled and randomized to one of the two groups will be included.

The baseline characteristics and laboratory data will be presented as the means and standard deviations for continuous variables and as frequencies and percentages for categorical variables. The incidence of AKI will be compared between the two groups using a $\chi^{2}$ test. The differences in changes in SCr levels and in the eGFR will be analyzed using Student's $t$-test or the Mann-Whitney $U$ test. A value of $P<0.05$ will be considered statistically significant. All analyses will be performed using SPSS Statistics software V21.0 (IBM Corporation, Armonk, NY, USA).

\section{Ethical approval}

The study will be performed in accordance with the Declaration of Helsinki, as amended by the 64th World Medical Association General Assembly in 2013. All of the participants will provide written, informed consent stating that participation is voluntary and can be withdrawn at any time. This study was approved by the Institutional Review Board of the Seoul National University Bundang Hospital (B-1408/264-002). The trial protocol has been registered at http://www.clinicaltrials.gov (NCT02250872).

\section{Discussion}

Cisplatin is an effective anti-tumor drug whose clinical application is limited by its nephrotoxicity $[1,8]$. Conventional renoprotective measures in patients receiving cisplatin are not satisfactory. Many studies have investigated the effects of protective strategies targeting the molecular mechanisms of cisplatin toxicity. Although encouraging results have been found in in vitro and in vivo models, a lack of positive data in clinical trials has prevented the effective clinical application of these strategies $[1,15,16]$. DPP4 inhibitors, which are antidiabetic drugs, have been shown to protect various organs from injuries [19-29]. This trial is the first to investigate whether a DPP4 inhibitor protects the kidney from cisplatin-induced injury in humans.

Previous studies have reported that cisplatin-induced AKI occurred in 6-35\% of patients within 7 days after a single dose of cisplatin $[1,5,6,31]$ and resolved within 15 days [7]. The incidence of AKI in patients treated with a single dose of cisplatin was $14.4 \%$ at our institution. The average follow-up time was 17 days. However, the incidence of AKI would be higher than $14.4 \%$ if we 
considered renal function within 7 days after cisplatin treatment; we estimate that the incidence of AKI would be approximately $25 \%$. We tentatively suggest that treatment with a DPP4 inhibitor could result in a $60 \%$ reduction in the risk of AKI. The statistical power may be compromised if sample size is calculated using a onesided level instead of a two-sided level. However, this trial is a phase II exploratory research, not a phase III or IV study on the possibility of direct clinical application. In addition, from the previous study [29], it is expected that DPP4 inhibitors will ameliorate rather than have no impact on or exacerbate cisplatin-induced AKI. It is not feasible to enroll more participants in our institution even though this study will be extended. Nevertheless, based on our assumptions, the sample size required for this study is larger than that of previous clinical studies of cisplatin-induced nephrotoxicity [15, 34-38].

We have considered the potential interactions between gemigliptin and cisplatin from the beginning of this study. First, cisplatin has no effect on hepatic cytochrome P450 3A4 (CYP3A4) in human liver microsomes [39], while gemigliptin is largely metabolized by CYP3A4 [40]. Second, cisplatin is mainly excreted by the kidney, and its nephrotoxicity is mediated via organic cation transporter 2 (OCT2) in the kidney [1, 41]. Gemigliptin and its active metabolite, LC15-0636, are very weak inhibitors of OCT2 in cultured xenopus oocytes. In terms of metabolic pathways and the renal transport system, cisplatin and gemigliptin never interact. Therefore, the renoprotective effect of gemigliptin could be investigated without affecting the anti-tumor activity of cisplatin.

The dose of gemigliptin in this study is two times higher than the dose used in the treatment of diabetes. Based on previous studies $[29,42]$, we determined that this dose was necessary to inhibit DPP4 activity by more than $80 \%$ during cisplatin exposure.

DPP4 inhibition has not caused hypoglycemia in studies of healthy volunteers $[43,44]$. Because the effects of GLP-1 on insulin secretion are glucose-dependent, the risk of hypoglycemia associated with DPP4 inhibitor treatment is low [45]. Nonetheless, we will exclude the participants with diabetes from this study. Therefore, the risk of hypoglycemia due to gemigliptin administration may be almost negligible in this trial.

A strength of this trial is that we will standardize hydration protocols before, during, and after cisplatin treatment to minimize the effects of confounding variables associated with renal outcomes. Cisplatin dosage per body surface area and the presence of diabetes have been reported to be the risk factors for moderate and severe cisplatin-induced AKI [31]. Another strength of this study is that randomization will be stratified on the basis of cisplatin dosage. Furthermore, participants with diabetes will be excluded from this study.
A small number of trials have investigated the clinical use of DPP4 inhibitors in the treatment of conditions other than diabetes. Treatments with DPP4 inhibitors have been investigated for their ability to enhance engraftment following umbilical cord blood transplantation in adults with hematologic malignancies [46], to improve endothelial dysfunction, and to prevent major cardiovascular events in patients with type 2 diabetes [47-49]. Again, we emphasize the originality of this trial, which will investigate the effect of a DPP4 inhibitor on cisplatin-induced AKI.

A limitation of this study is the diversity among the study participants and chemotherapeutic agents. Patients with different types of cancer receiving various anticancer drugs will be enrolled in this study. We expect that patients with gastric, biliary, head and neck, and lung cancer will be enrolled in this study. The following chemotherapeutic agents are used together with cisplatin in the treatment of these cancers: capecitabine, tegafur, docetaxel, pemetrexed, etoposide, irinotecan, and gemcitabine. All of these agents have been reported to have a low risk of AKI [50].

In summary, the present study is the first prospective, randomized controlled trial to evaluate the effect of a DPP4 inhibitor on cisplatin-induced AKI. The aim of this study is to demonstrate whether treatment with a DPP4 inhibitor, gemigliptin, can reduce the incidence of cisplatin-induced AKI in patients receiving cisplatin.

\section{Trial status}

This trial is ongoing. Participants are currently being recruited.

\section{Abbreviations \\ AKI: Acute kidney injury; DPP4: Dipeptidyl peptidase-4; GLP-1: Glucagon-like peptide-1; NSAID: Nonsteroidal anti-inflammatory drug; SCr: Serum creatinine; eGFR: Estimated glomerular filtration rate; PP: Per protocol; ITT: Intention-to treat; CYP3A4: Cytochrome P450 3A4; OCT2: Organic cation transporter.}

\section{Competing interests}

We declare that we have no competing interests.

\section{Authors' contributions}

SHB participated in the design of the study and drafted the manuscript. SHK, $J W K, Y J K$, and KWL participated in the design of the study and the data collection. KYN conceived the study, participated in acquiring funding, and had final responsibility for the decision to submit this manuscript for publication. All authors read and approved the final submitted manuscript.

\section{Acknowledgments}

The trial is funded by LG Life Sciences Seoul, Korea. Gemigliptin will be provided by the same pharmaceutical company for all participants. The sponsor of this study had no role in the study design, data collection, data review, data analysis, or report writing.

\section{Author details}

'Division of Nephrology, Department of Internal Medicine, Seoul National University Bundang Hospital, 82, Gumi-ro 173 Beon-gil, Bundang-gu, Seongnam, Gyeonggi-do 463-707, South Korea. ${ }^{2}$ Division of Hematology and Medical Oncology, Department of Internal Medicine, Seoul National 
University Bundang Hospital, 82, Gumi-ro 173 Beon-gil, Bundang-gu, Seongnam, Gyeonggi-do 463-707, South Korea.

\section{Received: 14 January 2015 Accepted: 21 May 2015 Published online: 29 May 2015}

\section{References}

1. Dos Santos NA, Carvalho Rodrigues MA, Martins NM, Dos Santos AC. Cisplatin-induced nephrotoxicity and targets of nephroprotection: an update. Arch Toxicol. 2012;86(8):1233-50.

2. Ozols RF, Young RC. High-dose cisplatin therapy in ovarian cancer. Semin Oncol. 1985;12(4 Suppl 6):21-30.

3. Sutton RA, Walker VR, Halabe A, Swenerton K, Coppin CM. Chronic hypomagnesemia caused by cisplatin: effect of calcitriol. J Lab Clin Med. 1991;117(1):40-3.

4. Jackson AM, Rose BD, Graff LG, Jacobs JB, Schwartz JH, Strauss GM, et al. Thrombotic microangiopathy and renal failure associated with antineoplastic chemotherapy. Ann Intern Med. 1984;101(1):41-4

5. Lebwohl D, Canetta R. Clinical development of platinum complexes in cancer therapy: an historical perspective and an update. Eur J Cancer. 1998;34(10):1522-34.

6. Wainford RD, Weaver RJ, Hawksworth GM. The immediate early genes, c-fos, C-jun and AP-1, are early markers of platinum analogue toxicity in human proximal tubular cell primary cultures. Toxicol In Vitro. 2009;23(5):780-8.

7. Gaspari F, Cravedi P, Mandala M, Perico N, De Leon FR, Stucchi N, et al. Predicting cisplatin-induced acute kidney injury by urinary neutrophil gelatinase-associated lipocalin excretion: a pilot prospective case-control study. Nephron Clin Pract. 2010;115(2):c154-60.

8. Leu L, Baribeault D. A comparison of the rates of cisplatin (CDDP)-induced nephrotoxicity associated with sodium loading or sodium loading with forced diuresis as a preventative measure. J Oncol Pharm Pract. 2010;16(3):167-71.

9. Lin HY, Lee SC, Lin SF, Hsiao HH, Liu YC, Yang WC, et al. Urinary neutrophil gelatinase-associated lipocalin levels predict cisplatin-induced acute kidney injury better than albuminuria or urinary cystatin C levels. Kaohsiung J Med Sci. 2013;29(6):304-11.

10. Yokoo S, Yonezawa A, Masuda S, Fukatsu A, Katsura T, Inui K. Differential contribution of organic cation transporters, OCT2 and MATE1, in platinum agent-induced nephrotoxicity. Biochem Pharmacol. 2007;74(3):477-87.

11. Choi MK, Song IS. Organic cation transporters and their pharmacokinetic and pharmacodynamic consequences. Drug Metab Pharmacokinet. 2008;23(4):243-53.

12. Luke DR, Vadiei K, Lopez-Berestein G. Role of vascular congestion in cisplatin-induced acute renal failure in the rat. Nephrol Dial Transplant. 1992;7(1):1-7.

13. Ramesh G, Reeves WB. p38 MAP kinase inhibition ameliorates cisplatin nephrotoxicity in mice. Am J Physiol Renal Physiol. 2005;289(1):F166-74.

14. Ramesh G, Reeves WB. TNF-alpha mediates chemokine and cytokine expression and renal injury in cisplatin nephrotoxicity. J Clin Invest. 2002;110(6):835-42.

15. Mousavi SS, Zadeh MH, Shahbazian H, Khanzadeh A, Hayati F, Ghorbani A, et al. The protective effect of theophyline in cisplatin nephrotoxicity. Saudi J Kidney Dis Transpl. 2014;25(2):333-7.

16. Ali BH, Al Moundhri MS. Agents ameliorating or augmenting the nephrotoxicity of cisplatin and other platinum compounds: a review of some recent research. Food Chem Toxicol. 2006;44(8):1173-83.

17. Drucker DJ, Nauck MA. The incretin system: glucagon-like peptide-1 receptor agonists and dipeptidyl peptidase-4 inhibitors in type 2 diabetes. Lancet. 2006;368(9548):1696-705.

18. Lambeir AM, Durinx C, Scharpe S, De Meester I. Dipeptidyl-peptidase IV from bench to bedside: an update on structural properties, functions, and clinical aspects of the enzyme DPP IV. Crit Rev Clin Lab Sci. 2003;40(3):209-94.

19. Jungraithmayr W, De Meester I, Matheeussen V, Baerts L, Arni S, Weder W. CD26/DPP-4 inhibition recruits regenerative stem cells via stromal cellderived factor-1 and beneficially influences ischaemia-reperfusion injury in mouse lung transplantation. Eur J Cardiothorac Surg. 2012;41(5):1166-73.

20. Zhai W, Jungraithmayr W, De Meester I, Inci I, Augustyns K, Arni S, et al. Primary graft dysfunction in lung transplantation: the role of CD26/ dipeptidylpeptidase IV and vasoactive intestinal peptide. Transplantation. 2009;87(8):1140-6.
21. Noyan-Ashraf MH, Momen MA, Ban K, Sadi AM, Zhou YQ, Riazi AM, et al. GLP-1R agonist liraglutide activates cytoprotective pathways and improves outcomes after experimental myocardial infarction in mice. Diabetes. 2009;58(4):975-83.

22. Sauve M, Ban K, Momen MA, Zhou YQ, Henkelman RM, Husain M, et al. Genetic deletion or pharmacological inhibition of dipeptidyl peptidase-4 improves cardiovascular outcomes after myocardial infarction in mice. Diabetes. 2010;59(4):1063-73.

23. Lim S, Choi SH, Shin H, Cho BJ, Park HS, Ahn BY, et al. Effect of a dipeptidyl peptidase-IV inhibitor, des-fluoro-sitagliptin, on neointimal formation after balloon injury in rats. PLoS One. 2012;7(4), e35007.

24. Mega C, De Lemos ET, Vala H, Fernandes R, Oliveira J, Mascarenhas-Melo F, et al. Diabetic nephropathy amelioration by a low-dose sitagliptin in an animal model of type 2 diabetes (Zucker diabetic fatty rat). Exp Diabetes Res. 2011;2011:162092.

25. Liu WJ, Xie SH, Liu YN, Kim W, Jin HY, Park SK, et al. Dipeptidyl peptidase IV inhibitor attenuates kidney injury in streptozotocin-induced diabetic rats. J Pharmacol Exp Ther. 2012;340(2):248-55.

26. Groop PH, Cooper ME, Perkovic V, Emser A, Woerle HJ, Von Eynatten M. Linagliptin lowers albuminuria on top of recommended standard treatment in patients with type 2 diabetes and renal dysfunction. Diabetes Care. 2013;36(11):3460-8.

27. Glorie LL, Verhulst A, Matheeussen V, Baerts L, Magielse J, Hermans N, et al. DPP4 inhibition improves functional outcome after renal ischemiareperfusion injury. Am J Physiol Renal Physiol. 2012;303(5):F681-8.

28. Joo KW, Kim S, Ahn SY, Chin HJ, Chae DW, Lee J, et al. Dipeptidyl peptidase IV inhibitor attenuates kidney injury in rat remnant kidney. BMC Nephrol. 2013;14:98.

29. Katagiri D, Hamasaki Y, Doi K, Okamoto K, Negishi K, Nangaku M, et al. Protection of glucagon-like peptide-1 in cisplatin-induced renal injury elucidates gut-kidney connection. J Am Soc Nephrol. 2013;24(12):2034-43.

30. Levey AS, Stevens LA, Schmid CH, Zhang YL, Castro 3rd AF, Feldman HI, et al. A new equation to estimate glomerular filtration rate. Ann Intern Med. 2009;150(9):604-12.

31. Mizuno T, Ishikawa K, Sato W, Koike T, Kushida M, Miyagawa Y, et al. The risk factors of severe acute kidney injury induced by Cisplatin. Oncology. 2013;85(6):364-9.

32. Mizuno T, Sato W, Ishikawa K, Shinjo H, Miyagawa $Y$, Noda $Y$, et al. KDIGO (Kidney Disease: Improving Global Outcomes) criteria could be a useful outcome predictor of cisplatin-induced acute kidney injury. Oncology. 2012;82(6):354-9.

33. Section 2: AKI Definition. Kidney Int Suppl (2011) 2012, 2(1):19-36.

34. Ghorbani A, Omidvar B, Parsi A. Protective effect of selenium on cisplatin induced nephrotoxicity: A double-blind controlled randomized clinical trial. J Nephropathol. 2013;2(2):129-34.

35. Hirosawa A, Niitani H, Hayashibara K, Tsuboi E. Effects of sodium thiosulfate in combination therapy of cis-dichlorodiammineplatinum and vindesine. Cancer Chemother Pharmacol. 1989;23(4):255-8.

36. Sleijfer DT, Offerman JJ, Mulder NH, Verweij M, van der Hem GK, Schraffordt Koops HS, et al. The protective potential of the combination of verapamil and cimetidine on cisplatin-induced nephrotoxicity in man. Cancer. 1987;60(11):2823-8.

37. Benoehr P, Krueth P, Bokemeyer C, Grenz A, Osswald H, Hartmann JT. Nephroprotection by theophylline in patients with cisplatin chemotherapy: a randomized, single-blinded, placebo-controlled trial. J Am Soc Nephrol. 2005;16(2):452-8.

38. Santoso JT, Lucci 3rd JA, Coleman RL, Schafer I, Hannigan EV. Saline, mannitol, and furosemide hydration in acute cisplatin nephrotoxicity: a randomized trial. Cancer Chemother Pharmacol. 2003;52(1):13-8.

39. Baumhakel M, Kasel D, Rao-Schymanski RA, Bocker R, Beckurts KT, Zaigler M, et al. Screening for inhibitory effects of antineoplastic agents on CYP3A4 in human liver microsomes. Int J Clin Pharmacol Ther. 2001;39(12):517-28.

40. Noh YH, Lim HS, Jin SJ, Kim MJ, Kim YH, Sung HR, et al. Effects of ketoconazole and rifampicin on the pharmacokinetics of gemigliptin, a dipeptidyl peptidase-IV inhibitor: a crossover drug-drug interaction study in healthy male Korean volunteers. Clin Ther. 2012;34(5):1182-94.

41. Ciarimboli G, Ludwig T, Lang D, Pavenstadt $H$, Koepsell $H$, Piechota $H J$, et al. Cisplatin nephrotoxicity is critically mediated via the human organic cation transporter 2. Am J Pathol. 2005;167(6):1477-84.

42. Lim KS, Kim JR, Choi YJ, Shin KH, Kim KP, Hong JH, et al. Pharmacokinetics, pharmacodynamics, and tolerability of the dipeptidyl peptidase IV inhibitor 
LC15-0444 in healthy Korean men: a dose-block-randomized, double-blind, placebo-controlled, ascending single-dose. Phase I study Clin Ther. 2008;30(10):1817-30

43. Bergman AJ, Stevens C, Zhou Y, Yi B, Laethem M, De Smet M, et al. Pharmacokinetic and pharmacodynamic properties of multiple oral doses of sitagliptin, a dipeptidyl peptidase-IV inhibitor: a double-blind, randomized, placebo-controlled study in healthy male volunteers. Clin Ther. 2006:28(1):55-72.

44. Lim KS, Cho JY, Kim BH, Kim JR, Kim HS, Kim DK, et al. Pharmacokinetics and pharmacodynamics of LC15-0444, a novel dipeptidyl peptidase IV inhibitor, after multiple dosing in healthy volunteers. Br J Clin Pharmacol. 2009;68(6):883-90.

45. Weir GC, Mojsov S, Hendrick GK, Habener JF. Glucagonlike peptide I (7-37) actions on endocrine pancreas. Diabetes. 1989;38:338-42.

46. Farag SS, Srivastava S, Messina-Graham S, Schwartz J, Robertson MJ, Abonour $\mathrm{R}$, et al. In vivo DPP-4 inhibition to enhance engraftment of single-unit cord blood transplants in adults with hematological malignancies. Stem Cells Dev. 2013;22(7):1007-15.

47. Van Poppel PC, Netea MG, Smits P, Tack CJ. Vildagliptin improves endothelium-dependent vasodilatation in type 2 diabetes. Diabetes Care. 2011;34(9):2072-7

48. Patil HR, Al Badarin FJ, Al Shami HA, Bhatti SK, Lavie CJ, Bell DS, et al. Metaanalysis of effect of dipeptidyl peptidase- 4 inhibitors on cardiovascular risk in type 2 diabetes mellitus. Am J Cardiol. 2012;110(6):826-33.

49. Matsubara J, Sugiyama S, Akiyama E, Iwashita S, Kurokawa H, Ohba K, et al. Dipeptidyl peptidase-4 inhibitor, sitagliptin, improves endothelial dysfunction in association with its anti-inflammatory effects in patients with coronary artery disease and uncontrolled diabetes. Circ J. 2013;77(5):1337-44.

50. Lexi-Comp I, American Pharmaceutical A. Drug information handbook. 2014

\section{Submit your next manuscript to BioMed Central and take full advantage of:}

- Convenient online submission

- Thorough peer review

- No space constraints or color figure charges

- Immediate publication on acceptance

- Inclusion in PubMed, CAS, Scopus and Google Scholar

- Research which is freely available for redistribution 